\title{
PROTEOCEPHALUS FILICOLLIS (RUD. 1810) IN THE NETHERLANDS
}

\author{
by \\ J. J. WILLEMSE AND A. L. M. VELTMAN \\ Zoological Laboratory, University of Amsterdam
}

\section{INTRODUCTION}

Cestodes of fishes have received very little attention in the Netherlands. In view of this, we have examined the intestinal contents of a great number of fishes from several places in this country. The study shows that proteocephalid infection is fairly common in some species of freshwater fishes. The present account is based upon the examination of specimens parasitic in the vicinity of Amsterdam and Den Helder during 1960,1961 and the first months of 1962 . In the course of the examination 2412 sticklebacks were examined: 1360 Pungitius pungitius, 771 Gasterosteus aculeatus f. trachurus and 281 Gasterosteus aculeatus f. hemigymnus. The investigations were carried out to determine (a) the incidence; and (b) the correlation between period of year and stage of development reached of Proteocephalus filicollis (Rud. 1810).

MegGrtT (1914) and Hopkins (1959) record the existence of such a correlation between time of year and stage of maturity reached. These accounts were based upon the examination of specimens parasitic in the alimentary canal of Gasterosteus aculeatus. No information has hitherto been published with regard to specimens parasitic in Pungitius pungitius.

\section{MATERIAL AND METHODS}

The Pungitius pungitius and Gasterosteus aculeatus f. hemigymnus were caught in the same ditches in the vicinity of Amsterdam. The Gasterosteus aculeatus $f$. trachurus were caught in canals at Den Helder. Fish were killed by decapitation and the abdomen was opened ventrally. The alimentary canal, from the stomach to the rectum inclusive, was removed and placed in a glass dish containing tap water. In most cases the canal was removed within 10 hours after the fish were captured. The intestine as well as the intestinal contents were examined under the dissection microscope, using $\times 30$ magnification. Many of the larger worms, when removed from the intestine, were seen discharging eggs. These eggs were used in experimentally infecting cyclops. They were pipetted in another glass dish containing about 50 specimens of Cyclops strenuus. A previous natural infection could be excluded. It was observed that the cyclops ingested eggs immediately after the eggs were added to the dish. Obviously the eggs attracted cyclops in one way or another. The dishes were kept at a temperature of $15-20^{\circ} \mathrm{C}$ and protozoan cultures were offered for feeding. Three weeks after the infective feed the cyclops were examined for parasitic infections under the microscope. It proved to be necessary to view the cyclops from its lateral as well as from its dorsal aspect. For that purpose they were rolled on the slide by moving the cover glass. Permanent preparations of worms were made by fixing in $10 \%$ formalin and staining with lactic carmin. Before fixation the worms were kept in tap water until no signs of life were exhibited.

\section{OBSERVATIONS}

Only three out of 281 specimens of Gasterosteus aculeatus f. hemigymnus were infected with Proteocephalus filicollis. No adult specimens were found. The most advanced stage was a worm showing genital primordia (November 1961). The fishes were caught in the months of January, February, March, April, July, October and November.

Gasterosteus aculeatus $f$. trachurus was not heavily infected either. In 10 out of 771 specimens worms were present. In most cases we found plerocercoids. Three fishes contained a mature worm each (MarchApril 1961). Dissections were carried out in February, March and April.

The number of infected Pungitius pungitius was considerably larger: 217 out of 1360 . In all months there was a considerable scatter in the degree to which development had proceeded. Fishes were caught in January, February, March, April, July, August, October, November and December.

It is of significance to indicate that all worms were carefully examined and compared with the description given by MegGrTt (1914). Without any doubt 

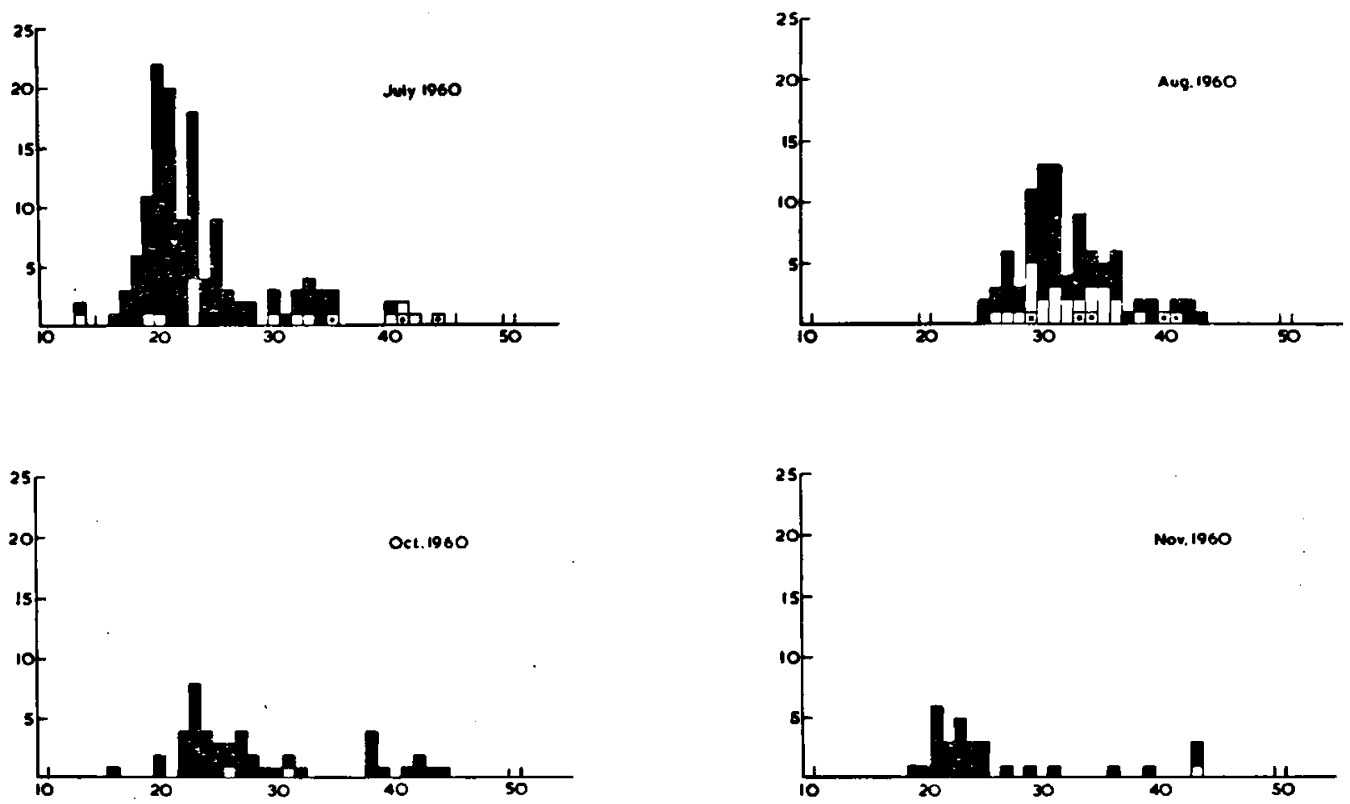

Fig. 1. The distribution of worms according to the size of the fish in four months of the year 1960 . The ordinate shows number of fish, the abcissa the length of fish in $\mathrm{mm}$. Black area shows number of uninfected fish; white the number of infected fish; white with black dot the number of fish infected with gravid worms.

they all are specimens of the species Proteocephalus filicollis.

Gravid worms from Pungitius pungitius did not exceed $19 \mathrm{~mm}$ in length. The length at which strobilation first occurs is $4 \mathrm{~mm}$. The three mature worms from Gasterosteus aculeatus $f$. trachurus measured 27, $22,14 \mathrm{~mm}$ in length. It is obvious that $P$. filicollis in Pungitius pungitius is considerably smaller than in Gasterosteus aculeatus.

In all cases the scolices of the worms were attached in the first part of the intestine, over $90 \%$ of all worms found had their scolices attached close to the pyloric valve. There appeared to be no correlation of position in the intestine with size of worm. Always the rectum was found to be devoid of worms.

The monthly distribution of Proteocephalus infections in Pungitius pungitius according to the size of the host fish is shown in Figs. 1 and 2. The results do not show a strongly marked seasonal cycle in the incidence. The histograms show that gravid worms are found in all months. In the months of March and April 1961 the larger fish are the most heavily infected. This higher incidence in the larger fish is less distinct in the other histograms. The March-April histograms 1961 also show a rise in incidence in general. It is obvious that both phenomena are abnormal.

In summer the young fish constitute an important part of the whole population. This is shown by the
July 1960 histogram. Even very young specimens are infected.

For each month we calculated an intensity index of the infection by dividing the total number of worms by the number of infected fish. The results showed little seasonal variation. The index fluctuated between 1.0 and 1.4. Most times 1.0 was found. The maximal number of worms in a fish was 5 .

No other helminth was found in the intestine of Pungitius pungitius and Gasterosteus aculeatus $f$, hemigymnus, while Gasterosteus aculeatus f. trachurus was infected with a number of other helminths. Podocotyle atomon was found in the intestine of 60 specimens, while 3 specimens were infected with Hemiurus sp. This trematode also lived in the intestine. Furthermore we found in the intestines of 140 fishes of the f. trachurus plerocercoids known as "Scolex polymorphus". These tetraphyllid larvae and the plerocercoids of Proteocephalus show a close resemblance in structure. In consequence it is possible that some of the worms parasitic in Gasterosteus aculeatus $f$. trachurus listed as Proteocephalus filicollis in fact are specimens of "Scolex polymorphus". Metacercariae were incapsulated in the liver and mesenteria of about 40\% of the dissected Pungitius. The dissections of both forms of Gasterosteus showed that these fishes were infected with metacercariae also.

Eggs obtained from gravid worms, found in the 

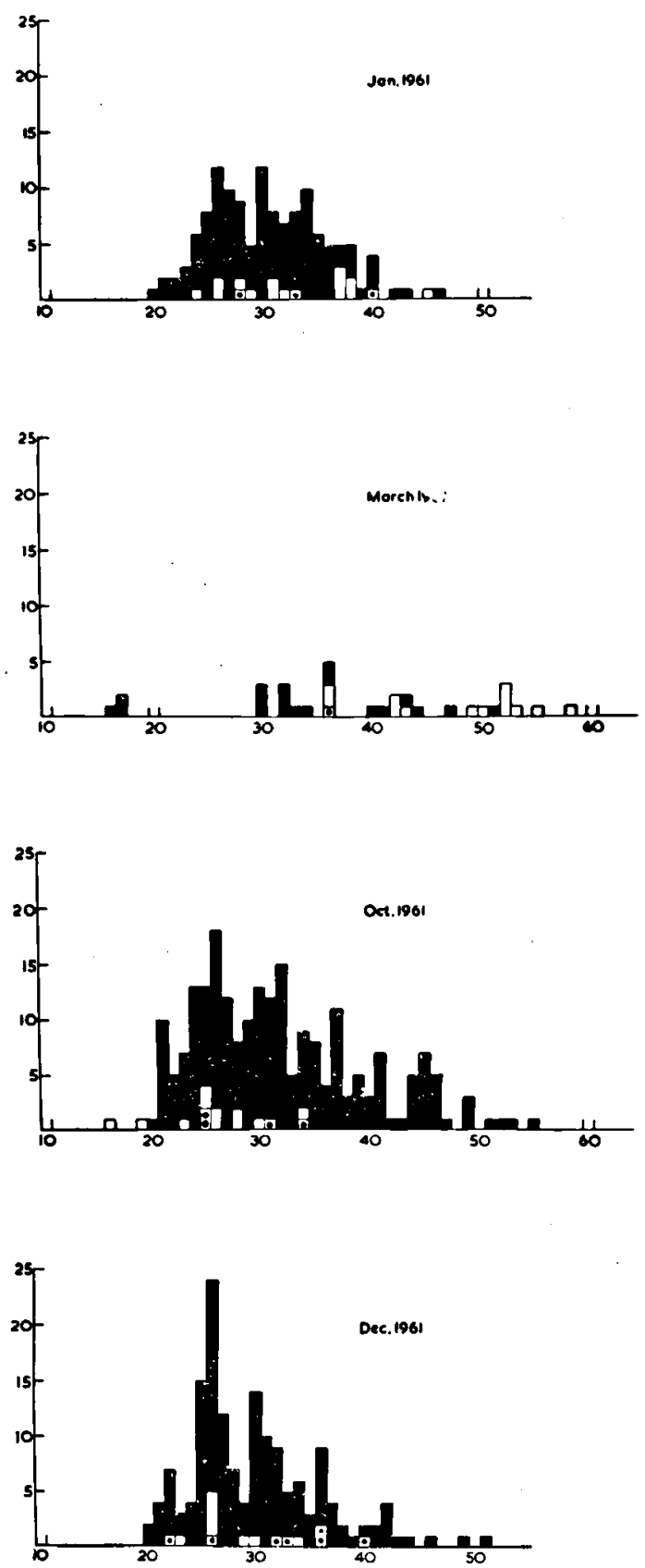
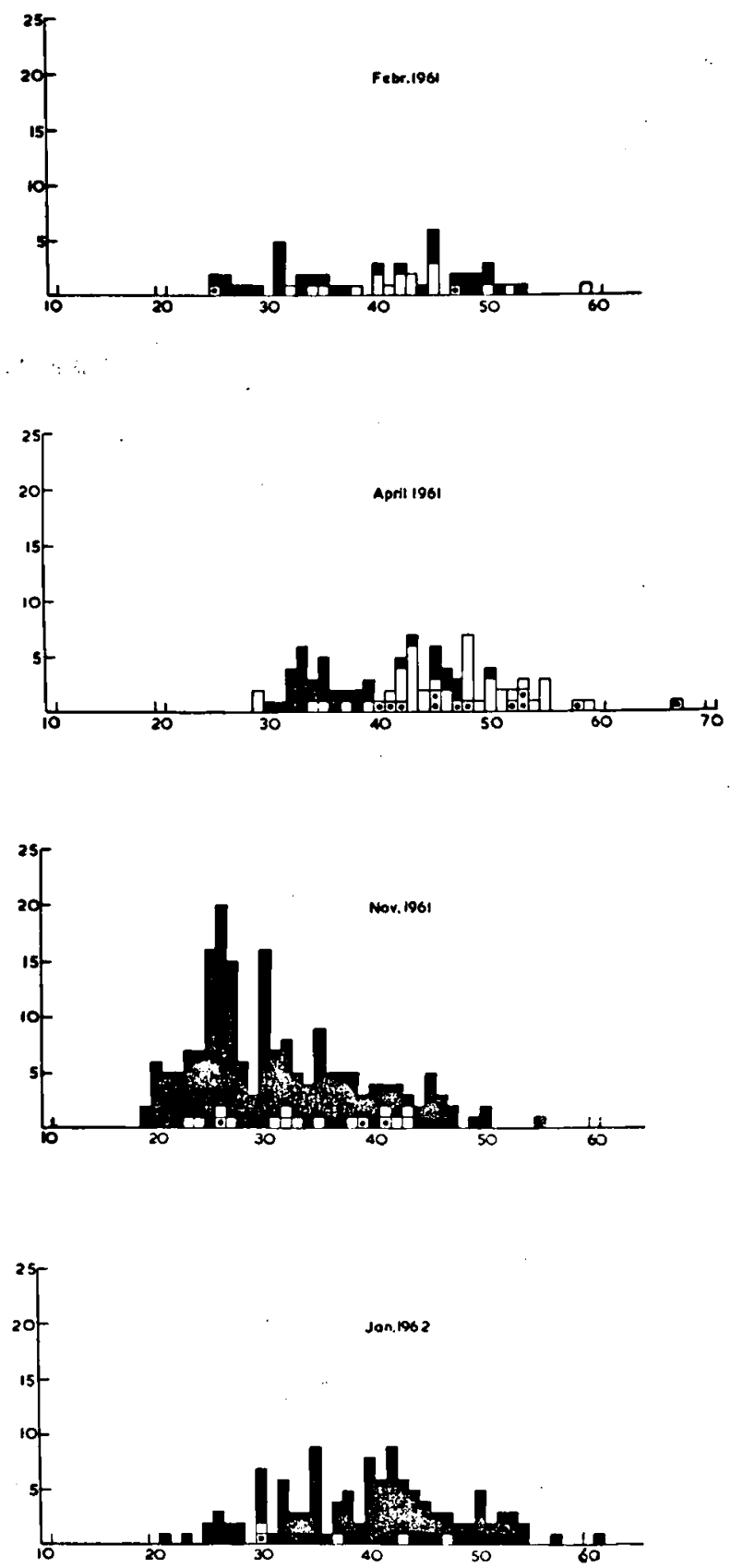

Fig. 2. The distribution of worms according to the size of the fish in 1961 and January 1962 . For explanation see fig. 1.

intestines of Pungitius pungitius were fed to cyclops in the months of July and August 1960; January, February, March, April, October, November and December 1961; January 1962. In all months procercoids were found in the cyclops. Several times infected cyclops thus obtained were fed to Pungitius pungitius. A previous infection could be excluded. Up to 48 plerocercoids were found in the intestine of a single fish a week after the infective feed. Three weeks after the infective feed the maximum number of worms found in the intestine of a single fish was 12. No extra-intestinal phase has been observed.

\section{DISCUSSION}

A correlation between time of year and stage of maturity reached has been shown to exist in several 
cestodes parasitic in freshwater fish of the temperate zone. According to the record of Hopkins (1959) in Proteocephalus filicollis, parasitic in Gasterosteus aculeatus, maturation shows a seasonal cycle. The histograms of Figs. I and 2 indicate that such a seasonal cycle does not exist in $P$. filicollis parasitic in Pungitius pungitius caught in the Netherlands. The presence of all stages of development and the presence of ripe eggs, infective to cyclops, in every season is an evidence that no annual cycle exists. Of course it is of particular interest to compare the different results obtained in North Lanarkshire and Holland. There can be little doubt that the checking of growth and development during winter observed by Hopkins is caused by a lowering of the temperature. If the temperatures in Scotland showed a more strongly marked annual cycle than in our country the different : esults would fit in very well with a temperature contral hypothesis as proposed by Hopkins. Unfortunately water temperatures were not available. Air temperatures recorded in Glasgow and De Bilt are given in the Table 1.

Table 1. Mean temperatures in ${ }^{\circ} \mathrm{C}$.

\begin{tabular}{|l|r|r|r|r|r|r|}
\hline & \multicolumn{3}{|c|}{ Glasgow } & \multicolumn{3}{c|}{ De Bilt } \\
\hline & 1956 & 1957 & 1958 & 1960 & 1961 & 1962 \\
\hline & & 4,4 & 2,4 & & 2,0 & 3,5 \\
Jan. & & 3,4 & 2,9 & & 6,3 & \\
Febr. & & 7,7 & 2,9 & & 7,1 & \\
March & & 8,5 & 7,2 & & 10,5 & \\
April & & 9,7 & 9,7 & & 11,0 & \\
May & & 13,9 & 12,7 & & 15,4 & \\
June & & 15,5 & 15,2 & 15,4 & 15,6 & \\
July & & 14,9 & 14,8 & 15,8 & 16,0 & \\
Aug. & & 10,7 & 14,5 & 13,6 & 16,6 & \\
Sept. & & 9,4 & & 10,8 & 11,4 & \\
Oct. & & 6,3 & & 7,5 & 4,7 & \\
Nov. & 5,6 & 6,3 & & 3,1 & 1,6 & \\
Dec. & 5,5 & 4,3 & & & & \\
& & & &
\end{tabular}

We do not believe that the differences shown in this table result in water temperatures that cause the existence of an annual cycle in North Lanarkshire and the lacking of an annual cycle in Holland. The differences of growth and development are obviously caused by host factors and they are not due to climatic factors.

Furthermore our investigations indicated that an annual cycle in the incidence level does not exist either. During our studies it was observed that worms are lost from the intestine of the fish host even when conditions are favourable. The simplest interpretation of these observations is that throughout the year an equilibrium exists between loss and gain. As ripe eggs were found in all months and adverse conditions such as shortage of food do not show a very strongly marked annual cycle in our country, both loss and gain are hardly seasonably dependent and loss equals gain throughout the year.

As already pointed out by MEgGrTr (1914) and Hopkins (1959) the procercoid of P.filicollis ingested in infected cyclops does not penetrate the intestine wall. The plerocercoid attaches itself to the intestine wall by means of its suckers. The phenomenon of initial attachment in the rectum, followed by a forward migration prior to maturation, recorded by Hopkins was not observed in our fish, neither in Pungitius pungitius nor in Gasterosteus aculeatus. It is probable, however, that the place of attachment is affected by the time needed for the digestion of the food (intermediate host). The place where the procercoids are released will depend on the time needed for the penetration of the cuticle of the intermediate host. It seems peculiar that the plerocercoids will show a preference for the rectum as a site of attachment.

There is no evidence for the existence of an extraintestinal phase of the life cycle in the fish host. Procercoids were released in the intestine and after three weeks they still did not penetrate the gut wall. Obviously they develop through the plerocercoid stage to the adults in the intestine of the fish.

Striking is the difference in incidence in Pungitius pungitius and Gasterosteus aculeatus f. hemigymnus. Both species were always caught in the same hauls. It is extremely improbable that $G$. aculeatus is an abnormal host. It seems more probable that the intermediate host is more frequently eaten by $P$. pungitius than by $G$. aculeatus, while a very small number of infected copepods are available. Very seldom cyclops were found in the intestinal contents of dissected fishes.

\section{SUMMARY}

The incidence of Proteocephalus filicollis in Pungitius pungitius, Gasterosteus aculeatus f. hemigymnus and Gasterosteus aculeatus $f$. trachurus has been studied. It is very low in both forms of Gasterosteus, but $16 \%$ of Pungitius was infected. The monthly examination of minimally 27 and maximally 223 Pungitius pungitius indicates that an annual cycle exists neither in the incidence nor in genital development. It seems probable that Proteocephalus filicollis in Pungitius pungitius is less affected by low temperatures than worms of the same species in Gasterosteus aculeatus.

Reference is made to intercurrent helminth infections. 


\section{LITERATURE}

Hopxiss, C. A., 1959: Seasonal variations in the incidence and development of the Cestode Proteocephalus filicollis (Rud. 1810) in Gasterosteus aculeatus. Parasitology 49, 529.

MEGGrrt, F. J., 1914: The structure and life-history of a tapeworm (Ichthyotaenia filicollis Rud.) parasitic in the stickleback. Proc. Zool. Soc. London 34, 113.

Wagner, E. D., 1954: The life history of Proteocephalus tumidocollis Wagner, 1953 (Cestoda) in Rainbow Trout. J. Parasit. 40, 489. 